\title{
Management of artery occlusion with autologous stem cells
}

Hak Chol Ri ${ }^{1}$, Yong Jun Pak ${ }^{1}$, Kyong Su Son ${ }^{1}$, Song Zu An ${ }^{1}$, Chang Ryong Pak ${ }^{1}$, Chol Ung Ri ${ }^{1}$, Chol Nam Pak ${ }^{1}$, Dae Chol Ri ${ }^{1}$

${ }^{1}$ Cloning Center, Biology Branch, Academy of Sciences, Pyong Yang, DPRKorea

Artery occlusion results in very serious gangrene in the legs and hands. There are some reports that MSCs and hematopoietic stem cells can make new blood vessel around ischemic lesions. We made animal models (rabbits) which have leg gangrene.

We tired Art. Femoralis in the rabbit's legs to block the blood supports for the legs.

After some days, surface temperature of the several parts of the legs began to be lower until $35.0{ }^{\circ} \mathrm{C}$ from the 39 ${ }^{\circ} \mathrm{C}$ and gangrene parts appeared. Human autologous stem cells were separated from the bone marrow of osiliac and used instantly or after culture. In the culture group MSCs were isolated by double direct adherence method instead of density separation. Briefly, after collecting the $20 \sim 50 \mathrm{ml}$ bone marrow, they were diluted (1:2) with PBS and centrifuged at $200 \mathrm{~g}$. Buffy coats were implanted in tissue culture flask. After $12 \mathrm{~h}$, the LDMEM with $15 \%$ Human cord blood serum (Home made) were changed and the cells in removed medium again were cultured instantly. After $24 \mathrm{~h}$, the medium were removed and second cells expanded more rapidly than the first one. The addition of removed old medium accelerated the expansion of the MSCs, even without growth factors.

About $2 \times 10^{9}$ nucleated cells from the human bone marrow or 4 30 million cultured human MSCs were injected into rabbit's legs around ischemic lesions. The surface temperature began to be higher and injury of the skin of the legs in model rabbits was getting better after two weeks. Clinical trials were carried out in 130 patients who were suffered from cryptogenic gangrene. After two weeks, the pain from the gangrene disappeared and regenerations were begun in the injuries in most of the patients. In the case of twice injection of stem cells the recovery efficacy was about $92.5 \%(74 / 80)$, but $73.3 \%(22 / 30)$ in single injection. In cultured MSCs case, however, there was no difference in the recovery rates in the range of 4 to 30 million MSCs numbers. In the recovered legs new blood vessels were identified with X-ray photographs. But the mechanism of the formation of the new blood vessel and the fates of the stem cells must be studied more.

Keywords: autologous stem cell, bone marrow, blood vessel

Cell Research (2008) 18:s82. doi: 10.1038/cr.2008.172; published online 4 August 2008

Correspondence: Hak Chol Ri

E-mail: pptayang@1@co.chesin.com 